\title{
Is first-line pembrolizumab appropriate for all patients with metastatic non-squamous histology non-small cell lung cancer patients?
}

\author{
Laura Le, Ritchell van Dams, Percy Lee \\ Department of Radiation Oncology, David Geffen School of Medicine at UCLA, Los Angeles, CA 90095, USA \\ Correspondence to: Percy Lee, MD. Department of Radiation Oncology, 200 UCLA Medical Plaza, Suite B265, Los Angeles, CA 90095, USA. \\ Email: percylee@mednet.ucla.edu. \\ Provenance: This is an invited article commissioned by the Section Editor Hengrui Liang (Department of Thoracic Surgery, Guangzhou Medical \\ University, Guangzhou, China). \\ Comment on: Gandhi L, Rodríguez-Abreu D, Gadgeel S, et al. Pembrolizumab plus chemotherapy in metastatic non-small-cell lung cancer. N Engl J \\ Med 2018;378:2078-92.
}

Submitted May 07, 2019. Accepted for publication May 20, 2019.

doi: 10.21037/tlcr.2019.05.11

View this article at: http://dx.doi.org/10.21037/tlcr.2019.05.11

Immune checkpoint inhibitors have emerged as a new frontier of modern cancer treatment. Prior to recent research in oncogenesis and the immune system, lung cancer treatment was mostly limited to platinum-based chemotherapy, radiation therapy, and surgical resection. Platinum-based chemotherapy has remained the standard of care for stage IV or metastatic non-small cell lung cancer (NSCLC) patients since a landmark meta-analysis in 1995, despite a relatively poor increase in median overall survival (OS) of 1.5 months (1). Incremental progress was seen with the addition of maintenance pemetrexed, but since 2009 the outcomes for patients with metastatic NSCLC without targetable driver mutations had stalled (2).

Research done at the beginning of this decade elucidated the mechanisms some lung tumors use to evade the immune system. Simply put, a cancer cell must exhibit a neoantigen or an overexpression of self-proteins to be recognized as abnormal by the immune system (3). Analysis of lung cancer specimens has shown that NSCLC tumor clonogens can escape the immune system by altering antigen presentation through disruption of the major histocompatibility complexes responsible for accurate detection and destruction by the host immune system (4). Additionally, lung tumors have been shown to inhibit cytotoxic $\mathrm{T}$ lymphocyte growth through increased binding of FOXP3+ T regulatory cells to activating cytokines, thus enhancing an immune-suppressing tumor microenvironment (5).
Tumor cells further override the endogenous immune system by inhibiting immune checkpoints. Programmed cell death receptor 1 (PD-1) is a protein primarily expressed by activated $\mathrm{B}$ cells, $\mathrm{T}$ cells, and NK cells, and regulates $\mathrm{T}$ cell activation in surrounding tissue (6). A major breakthrough occurred when it was shown that activation of PD-1 through the programmed cell death 1 ligand (PDL1) led to immunosuppression through diminished T-cell proliferation and reduced activity $(6,7)$. Knowledge of this pathway spurred intense interest in development of antiPD-1 and anti-PD-L1 antibodies for augmenting host antitumor immunity.

An early phase I study by Topalian et al. sought to determine the safety, efficacy and pharmacokinetics of nivolumab, a human IgG4-blocking monoclonal antiPD-1 antibody (8). Out of the 236 patients with advanced melanoma, NSCLC, prostate cancer or renal-cell/colorectal cancer, $18-28 \%$ of patients had an objective response to nivolumab with minimal adverse events. There was a PDL1 expression-dependent response, since an objective response was observed in $36 \%$ of $\mathrm{PD}-\mathrm{L} 1$-positive tumors and no response was seen for PD-L1-negative tumors (8). These promising results suggested the need to further explore PD-L1 as a cancer biomarker and unveiled the potential benefits of immune checkpoint inhibitors for oncologic treatment.

KEYNOTE-001 was the first clinical trial that explored 
the clinical implementation of pembrolizumab, another antiPD-1 antibody. This trial provided substantial supporting evidence for PD-L1 as a candidate biomarker in predicting response to anti-PD-1 therapies with pembrolizumab in the metastatic NSCLC setting. Garon et al. showed that PDL1 expression on NSCLC tumor cells (verified through immunohistochemistry) correlated to pembrolizumab response rates (9). In the validation cohort, patients with a PD-L1 tumor proportion score (TPS) $\geq 50 \%$ experienced an overall response rate (ORR) of $45.2 \%$ and median progression-free survival (PFS) of 6.3 months. However, among patients with TPS $<50 \%$ the ORR was $10.7-16.5 \%$ with a median PFS of approximately 4 months (9). KEYNOTE-001 demonstrated the clinical efficacy of pembrolizumab as an effective antitumor therapeutic and suggested that patients with metastatic NSCLC with TPS $\geq 50 \%$ potentially benefitted the most from this therapy.

The success of KEYNOTE-001 prompted several subsequent trials to determine the role of pembrolizumab in the treatment of patients with metastatic NSCLC. KEYNOTE-024 was an international, multicenter, phase III study that compared upfront pembrolizumab to standard chemotherapy for patients with $\geq 50 \%$ PDL1 expression (10). It found that within this population of patients with TPS $\geq 50 \%$, upfront pembrolizumab resulted in significantly longer PFS and OS compared to platinumbased chemotherapy [hazard ratio for death (HR), 0.60; $95 \%$ confidence interval (CI), 0.40-0.89; $\mathrm{P}=0.005)$. Patients treated with pembrolizumab also had an increased ORR compared to their chemotherapy-treated counterparts, $44.8 \%$ (95\% CI, 36.8-53.0\%) and $27.8 \%$ (95\% CI, $20.8-$ $35.7 \%$ ), respectively (10). Therefore, KEYNOTE-024 established upfront use of pembrolizumab and demonstrated its superiority compared to standard chemotherapy for patients with metastatic NSCLC with TPS $\geq 50 \%$ in the first-line setting.

Despite the promising results of KEYNOTE-024 for treatment of patients with PD-L1 expression $\geq 50 \%$, this cohort represents a minority of patients who present with metastatic NSCLC (9). Given the aggressive natural history of metastatic NSCLC with rapid disease progression, many patients never receive second-line treatment. KEYNOTE-189 was an international, multicenter, phase III trial of standard chemotherapy with or without pembrolizumab in patients with metastatic non-squamous histology NSCLC regardless of PD-L1 TPS (11). Patients were randomly assigned 2:1 to receive either (I) $200 \mathrm{mg}$ of pembrolizumab or (II) saline placebo, in addition to a platinum-based chemotherapy of the oncologist's choice. Pembrolizumab or the saline placebo were intravenously administered every 3 weeks for up to 35 cycles while simultaneously undergoing four cycles of chemotherapy. All patients had PD-L1 tumor proportion scores assessed by central laboratory review. Throughout the 126 sites in 16 countries, a total of 616 patients were enrolled. The trial demonstrated superior OS in the combination pembrolizumab arm, with an estimated 12-month OS of $69.2 \%$ (95\% CI, 64.1-73.8\%) compared to $49.4 \%$ (95\% CI, 42.1-56.2\%) in the chemotherapy with placebo group (HR for death $0.49,95 \%$ CI, 0.38-0.64, $\mathrm{P}<0.001$ ). The benefit of combination pembrolizumab was seen at all levels of PD-L1 expression. When subdivided by TPS, the greatest benefit was observed in patients with higher PDL1 expression levels: patients with $\geq 50 \%, 1-49 \%$, and $<1 \%$ tumor proportion scores having HRs for death of 0.42 ( $95 \%$ CI, 0.26-0.68), 0.55 (95\% CI, 0.34-0.90) and 0.59 (95\% CI, $0.38-0.92)$, respectively.

Similar advantages in PFS were observed for the combination pembrolizumab group, with a median PFS of 8.8 months (95\% CI, 7.6-9.2) in the combination pembrolizumab arm vs. 4.9 months (95\% CI, 4.7-5.5) for the chemotherapy with placebo group (HR for progression or death $0.52,95 \% \mathrm{CI}, 0.43-0.64, \mathrm{P}<0.001)$. PFS showed a similar PD-L1 TPS-dependent effect, as greater PDL1 expression levels correlated with longer progressionfree survival: patients with $\geq 50 \%, 1-49 \%$, and $<1 \%$ tumor proportion scores having HRs for disease progression or death of 0.36 (95\% CI, 0.25-0.52), 0.55 (95\% CI, 0.37-0.81) and 0.75 (95\% CI, 0.53-1.05), respectively.

Secondary end-points measured included objective response rate to the different combination therapies. As measured by blinded radiological review, the ORR was higher in the pembrolizumab-combination group than in the placebo-combination group across all categories of PD-L1 TPS. The ORR for the pembrolizumab group was 47.6\% (95\% CI, 42.6-52.5\%) vs. $18.9 \%$ (95\% CI, 13.8 $25.0 \%)$ in the chemotherapy-placebo group $(\mathrm{P}<0.001)(11)$.

KEYNOTE-189 marks an important milestone in the standardization of anti-PD-1 immunotherapy as first-line treatment for advanced NSCLC allowing greater access to more patients diagnosed with non-squamous histology NSCLC. This study identifies superior outcomes for combined pembrolizumab-chemotherapy in metastatic NSCLC regardless of PD-L1 TPS. This finding is best understood within the context of two similar trials exploring the use of pembrolizumab in advanced NSCLC. 
As previously discussed, KEYNOTE-024 established superiority of pembrolizumab monotherapy $v s$. standard chemotherapy for patients with $\geq 50 \%$ PD-L1 expression (10). For patients with rapidly progressive disease, combination pembrolizumab-chemotherapy likely provides added benefit via the rapid anti-tumor activity of platinumbased chemotherapy (12). It remains to be seen whether combination pembrolizumab-chemotherapy is superior to pembrolizumab alone in patients who present with relatively stable disease and high PD-L1 expression, and it is plausible that chemotherapy offers little more than excess toxicity in the first-line treatment of these patients.

The role for combination-pembrolizumab as firstline therapy in patients with $<50 \%$ PD-L1 expression is potentially confounded by the results of the recent KEYNOTE-042 trial. This international, multicenter, phase III trial randomized patients with locally advanced or metastatic NSCLC with PD-L1 TPS $>1 \%$ to either pembrolizumab alone or standard chemotherapy (13). It identified a significant improvement in OS for all patients, including those with 1-20\% PD-L1 expression (HR $0.81,95 \%$ CI, $0.71-0.93, \mathrm{P}=0.0018)$, most of the benefit remain in patients that have TPS $\geq 50 \%$. This result led to expansion of FDA approval of first-line pembrolizumab for "patients with stage III non-small cell lung cancer (NSCLC) who are not candidates for surgical resection or definitive chemoradiation or metastatic NSCLC" and PD-L1 TPS as low as $1 \%$ (14). Although the benefit of pembrolizumab monotherapy for patients with metastatic NSCLC with PD-L1 expression $\geq 1 \%$ is clear based on the results of KEYNOTE-024 and KEYNOTE-042, extreme caution must be exercised regarding patients with unresectable stage III NSCLC who are potentially curable with definitive chemoradiotherapy which remains an important therapy and part of the current standard-of-care in these patients. Pembrolizumab monotherapy has not been evaluated in a phase III trial against this current standard of care for these patients, definitive chemoradiation with consolidation durvalumab (15). Patient candidacy for surgical resection or chemoradiation should always be assessed on an individual basis by multidisciplinary oncological team.

In this context, perhaps the most important finding from KEYNOTE-189 is the demonstrated benefit to inclusion of pembrolizumab for patients with TPS $<1 \%$. This represents a paradigm shift in understanding the role of anti-PD-1 and anti-PD-L1 therapy for patients with zero or minimal upregulation of PD-L1, and it suggests the intriguing possibility that prior or concurrent therapy such as chemotherapy or radiotherapy could boost the host response to immune checkpoint inhibitors. The interplay between immunotherapy and radiotherapy is of particular interest, as ionizing radiation predominantly damages DNA and often results in an immunogenic cell death cascade $(16,17)$. Several preclinical murine models have revealed a synergy between high-dose stereotactic body radiotherapy (SBRT) and immune checkpoint inhibitors, whereby combined therapy is more efficacious than either administered alone (18-20).

Currently, there are a number of clinical trials evaluating the clinical application of combined immunotherapy and radiation treatment. Most ongoing trials are phase I safety and efficacy, but some notable phase II trials include the use of SBRT and anti-CTLA-4 (NCT02221739, New York University) and anti-PD-1 therapy (NCT02658097, Case Comprehensive Cancer Center). A few trials have reported promising early results in abstract form, including PEMBRO-RT which was a randomized phase II study of SBRT and pembrolizumab for metastatic NSCLC regardless of PD-L1 expression (21). This study randomized 74 patients with NSCLC on $\geq 2$ nd-line therapy and identified a notable extension of PFS to $6.4 v s .1 .8$ months for the SBRT-pembrolizumab arm vs. pembrolizumab alone (HR 0.55, 95\% CI, 0.31-0.98, P=0.04) (21). In addition, a secondary analysis of patients in the KEYNOTE-001 study identified a cohort of patients treated with pembrolizumab who had also received prior extracranial radiation. Although these patients received prior extracranial irradiation at a median 9.5 months prior to receipt of pembrolizumab, they had significantly longer PFS and overall survival (OS) compared to those who had received pembrolizumab alone and importantly there was no significant difference in grade $\geq 3$ pulmonary toxicity (22).

The encouraging, yet limited preclinical and clinical data from combining immunotherapy and radiation to target tumor growth reveals the need to further explore the molecular and clinical implication of this dual therapy. While immune checkpoint inhibition as monotherapy demonstrates clear benefit to patients with metastatic NSCLC and PD-L1 expression $\geq 1 \%$, the potential synergy with radiation should not be ignored as it provides the potential to expand and augment the response across an even larger subset of patients without detectable PD-L1 and help more patients diagnosed with NSCLC. 


\section{Acknowledgments}

None.

\section{Footnote}

Conflicts of Interest: Dr. Lee has received grant funding for a clinical trial, speaking honorarium, and travel reimbursement from AstraZeneca, Inc. The other authors have no conflicts of interest to declare.

\section{References}

1. Non-small Cell Lung Cancer Collaborative Group. Chemotherapy in non-small cell lung cancer: a metaanalysis using updated data on individual patients from 52 randomised clinical trials. BMJ 1995;311:899-909.

2. Ciuleanu T, Brodowicz T, Zielinski C, et al. Maintenance pemetrexed plus best supportive care versus placebo plus best supportive care for non-small-cell lung cancer: a randomised, double-blind, phase 3 study. Lancet 2009;374:1432-40.

3. Dunn GP, Old LJ, Schreiber RD. The Three Es of Cancer Immunoediting. Annu Rev Immunol 2004;22:329-60.

4. Rosenthal R, Cadieux EL, Salgado R, et al. Neoantigendirected immune escape in lung cancer evolution. Nature 2019;567:479-85.

5. Peng J, Yu Z, Xue L, et al. The effect of foxp3overexpressing Treg cells on non-small cell lung cancer cells. Mol Med Rep 2018;17:5860-8.

6. Zou W, Wolchok JD, Chen L. PD-L1 (B7-H1) and PD-1 pathway blockade for cancer therapy: Mechanisms, response biomarkers, and combinations. Sci Transl Med 2016;8:328rv4.

7. Pardoll DM. The blockade of immune checkpoints in cancer immunotherapy. Nat Rev Cancer 2012;12:252-64.

8. Topalian SL, Hodi FS, Brahmer JR, et al. Safety, Activity, and Immune Correlates of Anti-PD-1 Antibody in Cancer. N Engl J Med 2012;366:2443-54.

9. Garon EB, Rizvi NA, Hui R, et al. Pembrolizumab for the Treatment of Non-Small-Cell Lung Cancer. N Engl J Med 2015;372:2018-28.

10. Reck M, Rodríguez-Abreu D, Robinson AG, et al. Pembrolizumab versus Chemotherapy for PD-L1-Positive NonSmall-Cell Lung Cancer. N Engl J Med 2016;375:1823-33.

11. Gandhi L, Rodríguez-Abreu D, Gadgeel S, et al. Pembrolizumab plus Chemotherapy in Metastatic NonSmall-Cell Lung Cancer. N Engl J Med 2018;378:2078-92.

12. Lisberg A, Garon EB. Does Platinum-Based
Chemotherapy Still Have a Role in First-Line Treatment of Advanced Non-Small-Cell Lung Cancer? J Clin Oncol 2019;37:529-36.

13. Mok TSK, Wu Y, Kudaba I, et al. Pembrolizumab versus chemotherapy for previously untreated, PD-L1-expressing, locally advanced or metastatic non-small-cell lung cancer (KEYNOTE-042): a randomised, open-label, controlled, phase 3 trial. Lancet 2019;393:1819-30.

14. FDA. FDA expands pembrolizumab indication for firstline treatment of NSCLC (TPS $\geq 1 \%$ ). Available online: https://www.fda.gov/drugs/fda-expands-pembrolizumabindication-first-line-treatment-nsclc-tps-1

15. Antonia SJ, Villegas A, Daniel D, et al. Overall Survival with Durvalumab after Chemoradiotherapy in Stage III NSCLC. N Engl J Med 2018;379:2342-50.

16. Eriksson D, Stigbrand T. Radiation-induced cell death mechanisms. Tumor Biol 2010;31:363-72.

17. Galluzzi L, Maiuri MC, Vitale I, et al. Cell death modalities: classification and pathophysiological implications. Cell Death Differ 2007;14:1237-43.

18. Demaria S, Kawashima N, Yang AM, et al. Immunemediated inhibition of metastases after treatment with local radiation and CTLA-4 blockade in a mouse model of breast cancer. Clin Cancer Res 2005;11:728-34.

19. Park SS, Dong H, Liu X, et al. PD-1 Restrains Radiotherapy-Induced Abscopal Effect. Cancer Immunol Res 2015;3:610-9.

20. Twyman-Saint Victor C, Rech AJ, Maity A, et al. Radiation and dual checkpoint blockade activate non-redundant immune mechanisms in cancer. Nature 2015;520:373-7.

21. Theelen W, Lalezari F, de Vries J, et al. Randomized phase II study of pembrolizumab after stereotactic body radiotherapy (SBRT) versus pembrolizumab alone in patients with advanced non-small cell lung cancer: The PEMBRO-RT study. 2018 ASCO Annual Meeting Abstracts. Chicago: ASCO, 2018:9023.

22. Shaverdian N, Lisberg AE, Bornazyan K, et al. Previous radiotherapy and the clinical activity and toxicity of pembrolizumab in the treatment of non-small-cell lung cancer: a secondary analysis of the KEYNOTE-001 phase 1 trial. Lancet Oncol 2017;18:895-903.

Cite this article as: Le L, van Dams R, Lee P. Is first-line pembrolizumab appropriate for all patients with metastatic nonsquamous histology non-small cell lung cancer patients? Transl Lung Cancer Res 2019;8(Suppl 4):S327-S330. doi: 10.21037/ tlcr.2019.05.11 